\title{
Flower trade-offs derived from nectar investment in female reproduction of two Nicotiana species (Solanaceae)
}

\author{
Leonardo Galetto ${ }^{1,2 *}$ (D), Francielle Paulina Araujo ${ }^{3}$ (D), Gabriel Grilli' (D), Leonardo D. Amarilla ${ }^{1,2}$ (D), Carolina \\ Torres $^{1,2}$ (1) and Marlies Sazima ${ }^{4}$ (1)
}

Received: March 29, 2018

Accepted: July 13, 2018

\begin{abstract}
Animal-pollinated flowers may orient resources for competing activities, such as nectar production for attracting flower visitors but then saving nectar (through inhibiting nectar production or by final resorption) for the subsequent maturation of fruits and seeds. Nectar production is continuous in Nicotiana longiflora and N. alata after flower opening, but early nectar removal reduces total secreted nectar. Resource trade-off between nectar investment and seed production were experimentally assessed in manually pollinated flowers experiencing different numbers of repeated nectar removals, while controlling for maternal effects. We expected that flowers with less nectar secretion produce larger seed sets. The results showed that for both species the earlier the nectar removal during flower anthesis, the lower the total nectar secreted and the higher the mass of seeds produced. This general pattern was clearer for $N$. longiflora. The link between decreased nectar production and the subsequent increase in the seed set implies that resources are limited. Consequently, nectar savings during the pollination process through early nectar removal by pollinators can be interpreted as a trade-off between resources secreted by flowers for pollinator attraction and those utilized during fruit and seed maturation.
\end{abstract}

Keywords: nectar dynamics, nectar removal effects, nectar saving, Nicotiana alata, N. longiflora, seed production

\section{Introduction}

Flowers invest resources that may represent trade-offs between different physiological processes during their anthesis and fruit development. For example, flowers invest resources in maintenance processes such as respiration and transpiration, and in attracting pollinators through the production of pollen and nectar (Obeso 2002). Resource allocation during reproduction can be examined at different plant levels because of its modularity (Obeso 2004). For example, reproduction investment represents the integration of adjustments at different hierarchical levels within the plant. In particular, nectar dynamics can involve mechanisms to save nectar-energetic investments, and then derive resources to fruit and seed development. Nevertheless, the trade-offs of energetic investments between nectar (through nectar production, replenishment or resorption) and female reproduction depend on the plant-modular level at which they are analyzed (Obeso 2002; 2004).

Investment in nectar production was measured in some species and varies considerably; it represents $>30 \%$ of the floral resources in Asclepias (Pleasants \& Chaplin 1983;

1 Instituto Multidisciplinario de Biología Vegetal, Consejo Nacional de Investigaciones Científicas y Técnicas, Universidad Nacional de Córdoba, X5016GCA, Córdoba, Argentina

2 Cátedra de Diversidad Biológica III, Facultad de Ciencias Exactas, Físicas y Naturales, Universidad Nacional de Córdoba, X5016GCA, Córdoba, Argentina

3 Universidade Estadual do Rio Grande do Sul, 95400-000, São Francisco de Paula, RS, Brazil

4 Departamento de Biologia Vegetal, Instituto de Biologia, Universidade Estadual de Campinas, 13083-970, Campinas, SP, Brazil

* Corresponding author: leo@imbiv.unc.edu.ar 
Southwick 1984), while in other species, it represents only $3 \%$ as in Pontederia cordata (Harder \& Barrett 1992). When nectar production implies reproductive decreases in terms of male or female production, it may represent a trade-off (Pleasants \& Chaplin 1983; Obeso 2002). Nevertheless, experimental evidence did not show clear patterns to disentangle if nectar investments necessarily imply a subsequent seed set reduction. At the plant level, repeated nectar removals caused a significant reduction in seed number for Blandfordia nobilis (Pyke 1991), Clarkia tembloriensis (Ashman \& Schoen 1997) and Tillandsia multicaulis (Ordano \& Ornelas 2005). In contrast, nectar production did not show female reproductive effects in Asclepias quadrifolia (Pleasants \& Chaplin 1983), Polemonium foliosissimun (Zimmerman \& Pyke 1988), Prosopis glandulosa (Golubov et al. 2004), Echium vulgare (Leiss et al. 2004), and Tillandsia deppeana (Ordano \& Ornelas 2005).

In summary, animal-pollinated flowers may orient resources for competing activities, such as nectar production for attracting flower visitors but then saving nectar for the subsequent maturation of fruits and seeds. Consequently, reproduction may entails dynamic trade-offs between "gains" and "costs" of nectar investment for the different interacting components during flower anthesis and fruit maturation (Obeso 2002). Different processes of nectar physiology during flower anthesis can be conceptualized as nectar costs (e.g., nectar secretion) or as nectar gains (e.g., nectar resorption or nectar saving through inhibition after removals).

Nicotiana longiflora presents a continuous nectar secretion pattern through the anthesis (Galetto \& Bernardello 1993). Natural nectar production (in terms of volume and amount of solutes secreted by bagged flowers; i.e., with no pollinator visits) is increased as the flower ages, reaching the maximum values within the fourth day after opening. Also, flowers did not reabsorb nectar at the end of the flower lifetime. Nevertheless, when flowers suffer experimental removals (i.e., a simulated pollinator visit), nectar production is almost stopped. Consequently, flowers of this species strongly respond to nectar removals decreasing resource investments for nectar (Galetto \& Bernardello 1993). These characteristics in nectar secretion allow us to experimentally compare flowers with maximum nectar secretion (no removals) to flowers whose nectar was previously extracted at different flower-ages. The main hypothesis we propose is if animal-pollinated flowers save resources regulating nectar secretion according to the timing of the pollinator visit, the lower energy invested in nectar production (yet enough to achieve pollination) by earlyvisited flowers can entail a benefit for subsequent fruit and seed maturation. We want to test (at the flower/fruit modular level) if the total amount of nectar produced by the flower (as the indicator of the resource investment during the pollination process) affects seed set (as the indicator of the resource investment during the fruit/ seed maturation process). We expected that flowers with less nectar secretion produce larger seed sets than flowers secreting their maximum.

\section{Materials and methods}

\section{Studied species}

Two Nicotiana species belonging to the same taxonomic section Alatae (Goodspeed 1954) were used as models to test the possible energy trade-off between nectar investment and fruit maturation. These species are not clonal and pollination did not induce flower wilting. Nicotiana longiflora is a self-compatible (Primo \& Galetto 2001), ruderal species that can be found mainly distributed in Argentina and Paraguay (Goodspeed 1954) and pollinated by Sphingids (Galetto \& Bernardello 1993). We studied individuals from natural populations growing near Córdoba city, Argentina $\left(31^{\circ} 26^{\prime} 25^{\prime \prime} \mathrm{S} 64^{\circ} 11^{\prime} 30^{\prime \prime} \mathrm{W}\right)$. This is a perennial herbaceous species, with whitish flowers presenting a tubular corolla of 9-12 cm length and an extended flower anthesis of four days (Primo \& Galetto 2001). Nicotiana alata is distributed through southern Brazil, northeastern Argentina, and Paraguay (Goodspeed 1954). This species is self-incompatible with white, long-tubed flowers lasting five days, and visited by large hawk moths (Sphingidae) (Ippolito et al. 2004). We performed the experimental study with individuals under greenhouse conditions $\left(28^{\circ} \mathrm{C}\right.$ with $16 / 8 \mathrm{~h} \mathrm{light/dark} \mathrm{period).}$ Individuals of $N$. alata came from seeds collected in the

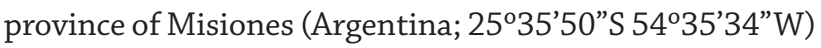
from a natural population described in Roldán et al. (2010).

\section{Nectar dynamics and reproductive trade-offs}

Ten plants of $N$. longiflora were examined under field conditions. Nectar measurements were performed in bagged inflorescences by following the flowers' development until the corollas began to wilt. Inflorescences with developed buds were covered using mesh bags to prevent pollinator visits. Four individual buds of each plant were tagged for identification; one flower per inflorescence was assigned for each experimental treatment (a total of 40 flowers, ten per treatment; see below). After the natural wilting of experimentally treated flowers, bags were removed from inflorescences during fruit maturation. Buds were always selected in the middle of the inflorescences to standardize treatments. Twenty individuals of $N$. alata growing under greenhouse conditions were selected and measured at two different periods of the year (10 individuals at each period; two individuals that presented disease signals were excluded during the second period). Five individual buds on each inflorescence were tagged for identification; one flower per inflorescence was assigned for each experimental treatment (a total of 90 flowers; see below). Plants were not bagged because under greenhouse conditions, pollinators cannot 
visit the flowers. The number and frequency of nectar measurements differed between species and treatments (see below).

The experimental design throughout the flower anthesis (i.e., a different number of nectar extractions to the same flower set) was as follows: a) the first set of flowers (set 5 or 4 for $N$. alata and $N$. longiflora, respectively) was drained five/ four times, the second (set 4 or 3 ) four/three times, and so on until set 1 when nectar was measured only once at the end of the anthesis (see details in Galetto \& Bernardello 2004). The untreated flowers on the inflorescences of both species were maintained. We measured the total accumulated nectar among flower sets experiencing different numbers of repeated nectar removals. We assumed that micro-syringe insertions have negligible effect on nectar production (see Ordano \& Ornelas 2005). The removals were performed at $24 \mathrm{~h}$ intervals during the flower lifespan. Nectar volume was measured using a graduated micro-syringe (Hamilton, NV, USA) without removing the flowers from the plant and taking extreme care to withdraw all nectar (i.e., avoiding nectary damage and accidental pollination when extracting nectar). Sugar concentration (percentage sucrose, mass / mass) was measured with a pocket refractometer $(0-32 \%$; Atago, Tokyo, Japan). The solutes per $\mu \mathrm{L}$ of nectar secreted by each flower were estimated with this equation: $y=0.00226$ $+(0.00937 x)+(0.0000585 x 2)$, where the value of $x$ is the concentration (i.e., the reading of the refractometer) and $y$ is the mg of solutes per $\mu \mathrm{L}$ (Galetto \& Bernardello 2005). The total solutes secreted by each flower were obtained multiplying $y$ by the total volume (Galetto \& Bernardello 2005).

All the treated flowers (i.e., flower sets experiencing different numbers of repeated nectar removals) were manually cross-pollinated during the second day of anthesis, controlling the amount of deposited pollen (each stigma was touched twice with a recently open anther from different individuals). Pollen deposition occurred during the stigmatic receptivity period and pollen loads were sufficient to fertilize ovules (see Primo \& Galetto 2001). Fruits and seeds from experimental flowers (all the treated flowers set fruits) were then collected. Fruits and seeds of each fruit were weighed (seed mass) to the nearest $0.001 \mathrm{mg}$. Results are presented as seed mass produced because seeds are numerous and minuscule. Samples of 50 seeds were weighed (to the nearest $0.0001 \mathrm{mg}$ ) to estimate the total seed number produced by fruit in these Nicotiana species (a mean of 990.8 seeds per fruit for $N$. alata, $\mathrm{n}=15$; and a mean of 402.9 seeds per fruit for $N$. longiflora, $\mathrm{n}=10$ ).

\section{Statistical analysis}

The effects of the different numbers of repeated nectar removals on nectar traits and the subsequent fruit and seed maturation were evaluated separately for each plant species. The response variables analyzed were 'nectar concentration', 'nectar volume' and 'nectar solutes' for nectar traits, and 'fruit mass' and 'seed mass' for plant fitness. We used linear mixed models (lme) from package nlme with "nectar removals" as fixed factor with 5 levels in $N$. alata and 4 levels in N. longiflora, and "individuals" as random block effect. The model selection procedure used in this study is based in the Akaike information criterion (AIC) that uses deviance as a measure of fit, adding a term to penalize more complex models (i.e. greater numbers of parameters). The lower AIC indicates a better trade-off between fit and complexity of the model. Rather than estimating $p$ values, informationtheoretical methods estimate statistics that quantify the magnitude of the difference between models in expected predictive power (Bolker et al. 2009). Therefore, model selection chooses the simplest model in the case where differences in the AIC were not significant under deviance analyses (Model S1 in supplementary material). The random block effect (i.e., "individuals") was not included in the simplest model with the lower AIC because differences in the deviance were not significant. Consequently, the simplest linear model (i.e. without the random term) was evaluated with the $\operatorname{lm}($ ) function from package stats. The post-hoc tests were performed with the pairwise.t.test() function from package stats to test the significant differences between treatments (Model S2 in supplementary material). All the analyses were conducted on R Core Team (2017).

\section{Results}

We confirmed the reported pattern for bagged flowers of N. longiflora (Galetto \& Bernardello 1993) on the natural nectar dynamics throughout the anthesis for both species. Summarizing, nectar secretion was increased and nectar concentration was stable through flower anthesis of these two Nicotiana species; no nectar resorption was detected at the end of the flower lifetime (data not shown). Consequently, increases in the content of total nectar solutes were observed as flower ages (data not shown).

Both Nicotiana species showed a relatively constant nectar concentration throughout flower anthesis independently of the number of removals performed to each flower set (the differences were not significant among flower sets; Fig. 1). Conversely, total nectar produced was lower in those flowers drained earlier (i.e., flower sets experiencing a higher number of nectar withdraws; data for total volume and solutes in Figs. 2 and 3, respectively). In the case of N. alata, only the total nectar produced by the flower set experiencing the maximum number of removals (set 5) was significantly different from the others in terms of total volume (Fig. 2) and solutes (Fig. 3). The results in N. longiflora showed that the earlier the nectar removal during flower anthesis (i.e., set 4 and 3), the lower the total nectar secreted in terms of volume or solutes (Figs. 2 and 3, respectively).

The differences in the total nectar production by flower sets experiencing repeated nectar removals affected fruit 


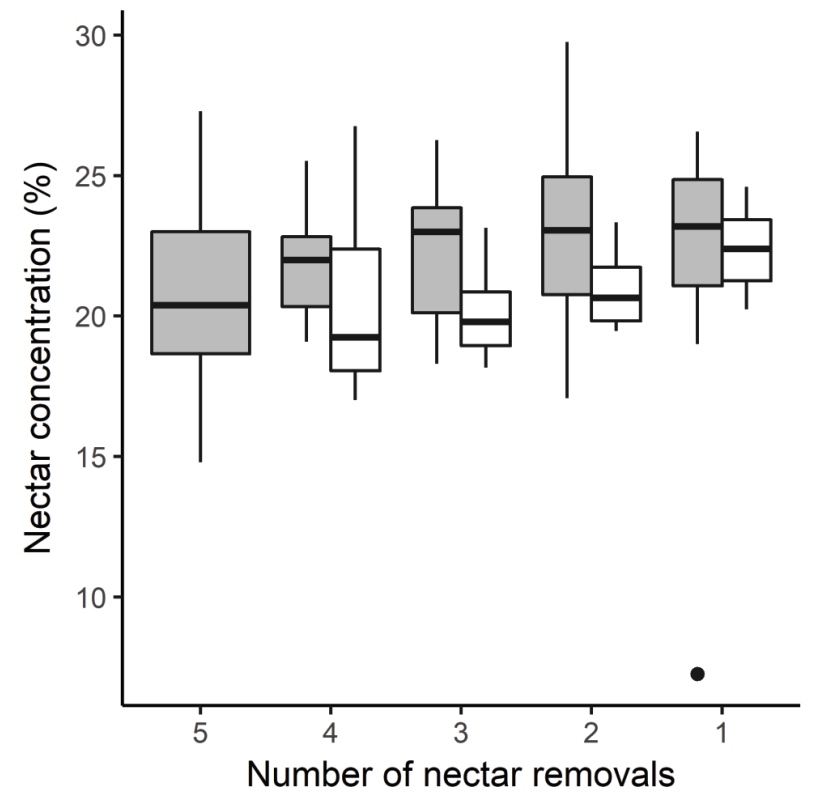

Figure 1. Removal effects on nectar concentration (\%; mass. mass $^{-1}$ ) of Nicotiana alata (grey) and N. longiflora (white) flower sets (5 and 4 respectively) with different number of withdrawals $\left(\mathrm{F}_{[4,82]}=1.22, \mathrm{P}=0.31\right.$ and $\mathrm{F}_{[3,36]}=2.73, \mathrm{P}=0.058$, respectively $)$ ordered from the earliest (5) to the last one (1) at the end of the flower lifetime. Boxplot represents: median (medium line), first and third quartile (box), minimum and maximum values (whiskers) and outliers (dots).

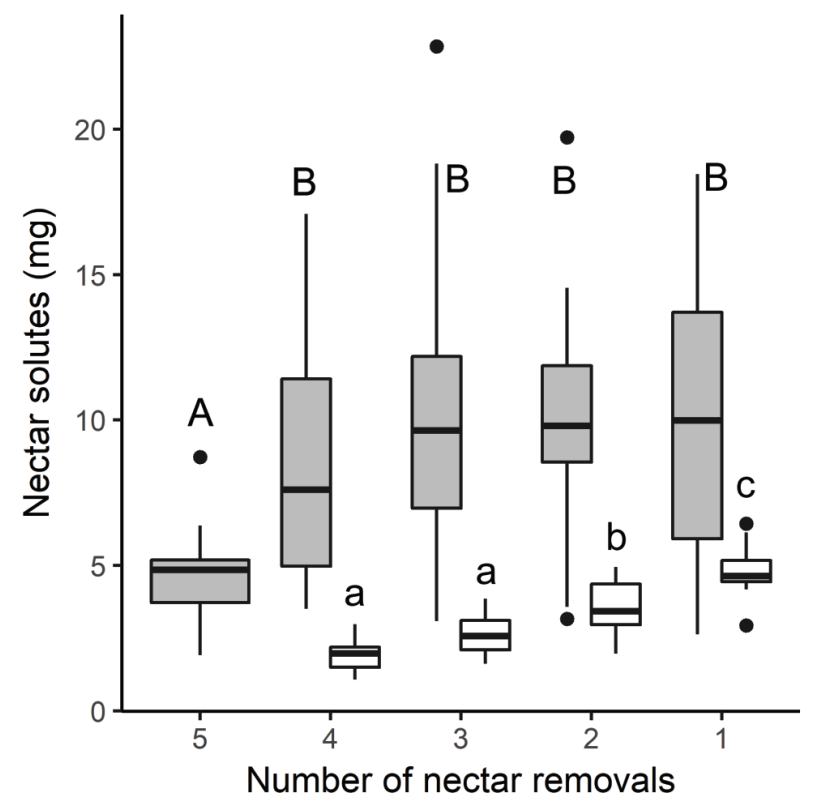

Figure 3. Removal effects on mass of solutes (mg of nectar solutes per flower) of Nicotiana alata (grey) and N. longiflora (white) flower sets (5 and 4 respectively) with different number of withdrawals $\left(\mathrm{F}_{[4,82]}=5.26, \mathrm{P}=0.0008\right.$ and $\mathrm{F}_{[3,36]}=23.5, \mathrm{P}$ $<0.0001$, respectively) ordered from the earliest (5) to the last one (1) at the end of the flower lifetime. Boxplot represents: median (medium line), first and third quartile (box), minimum and maximum values (whiskers) and outliers (dots).

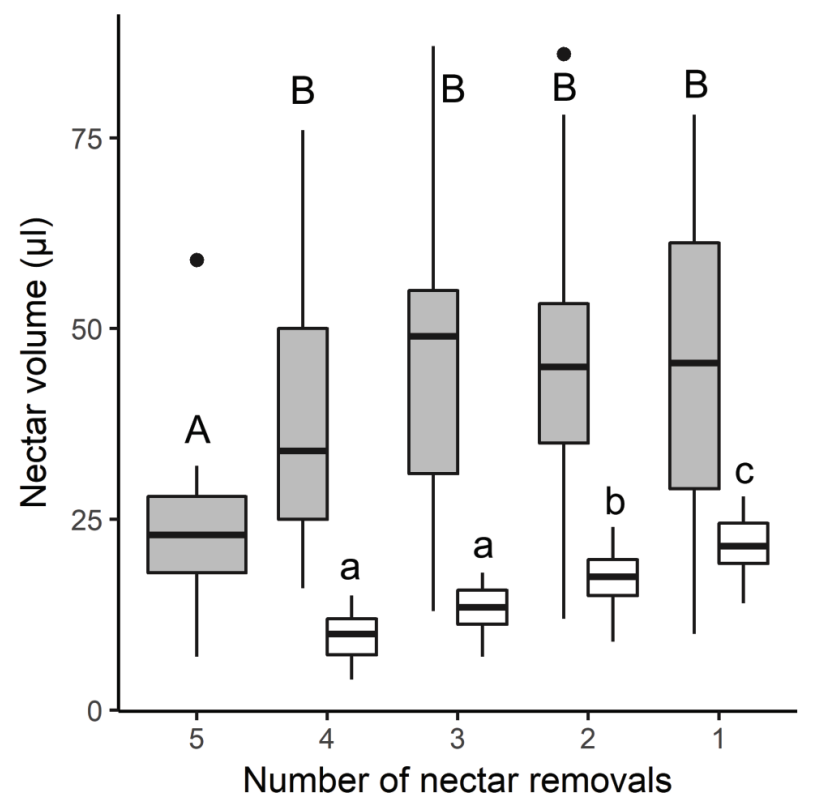

Figure 2. Removal effects on nectar volume ( $\mu$ l per flower) of Nicotiana alata (grey) and N. longiflora (white) flower sets (5 and 4 respectively) with different number of withdrawals $\left(\mathrm{F}_{[4,82]}=4.12\right.$, $\mathrm{P}=0.004$ and $\mathrm{F}_{[3,36]}=16.39, \mathrm{P}<0.0001$, respectively) ordered from the earliest (5) to the last one (1) at the end of the flower lifetime. Boxplot represents: median (medium line), first and third quartile (box), minimum and maximum values (whiskers) and outliers (dots).

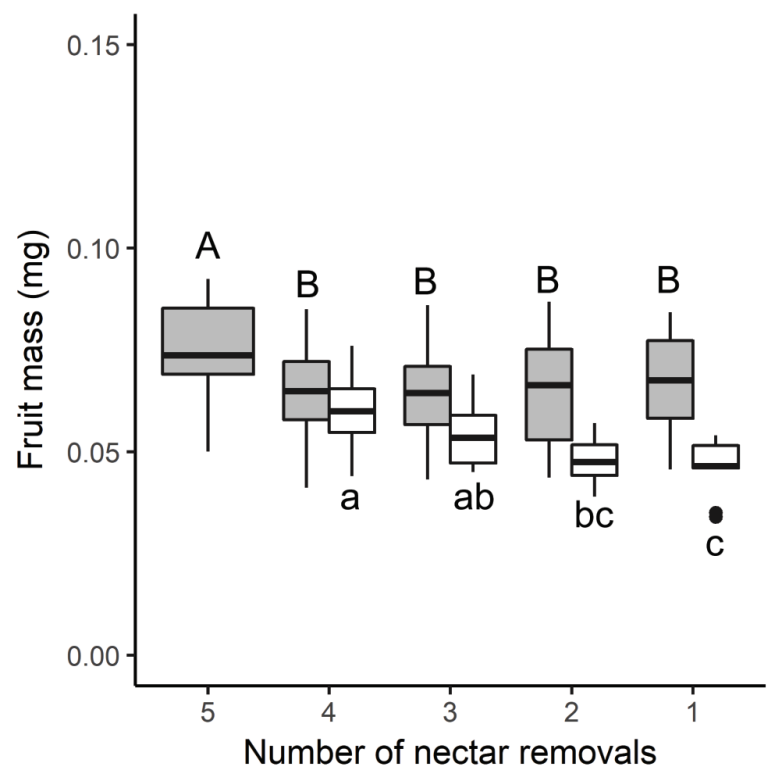

Figure 4. Nectar removal effects on total fruit mass (mg of complete fruits) of Nicotiana alata (grey) and N. longiflora (white). Fruits were obtained from equally pollinated flower sets ( 5 and 4 respectively) previously subjected to a different number of nectar withdrawals $\left(\mathrm{F}_{[4,82]}=2.72, \mathrm{P}=0.035\right.$ and $\mathrm{F}_{[3,36]}=6.2, \mathrm{P}=0.02$, respectively) ordered from the earliest (5) to the last one (1) at the end of the flower lifetime. Boxplot represents: median (medium line), first and third quartile (box), minimum and maximum values (whiskers) and outliers (dots). 
and seed production in both Nicotiana species; in particular, higher nectar saving showed a positive effect on the reproductive output in both species (Figs. 4, 5). Fruit and seed mass were maximum in those flower sets with the higher number of removals (i.e., removed earlier with the higher nectar saving; Figs. 4 and 5). In the case of $N$. alata, only the flower set subjected to the maximum number of removals (set 5) was significantly different from the others in terms of fruit (Fig. 4) and seed mass (Fig. 5). N. longiflora showed a clearer pattern for seed mass than for fruit mass (Figs. 4 and 5). Seed mass was higher in those flower sets experiencing earlier nectar removals (i.e., set 4 or 3; Fig. 5) compared to those removed later (sets 2 and 1; Fig. 5).

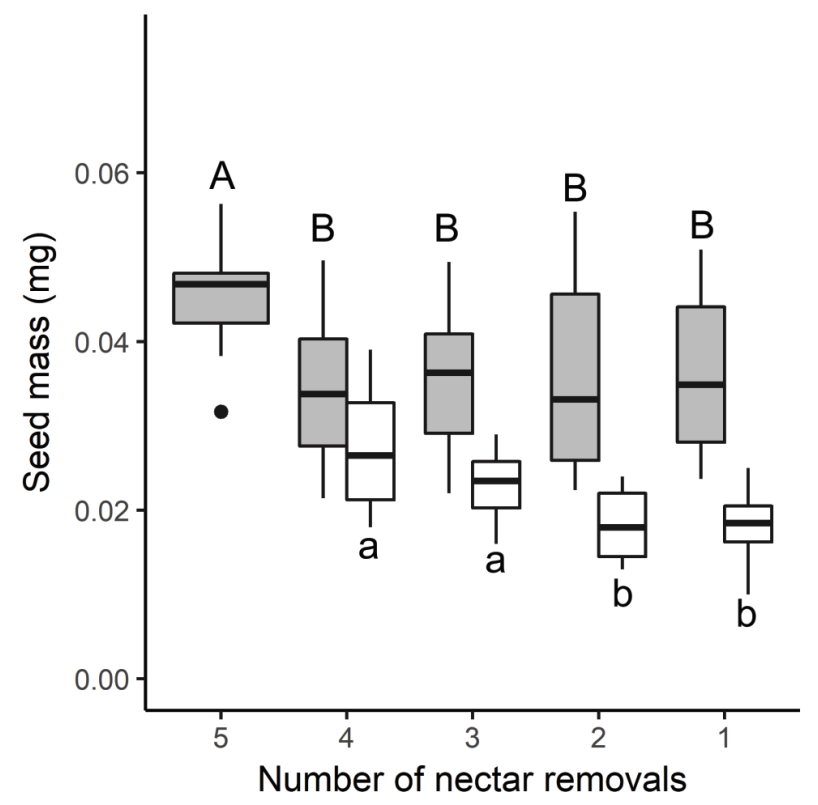

Figure 5. Nectar removal effects on total seed mass (mg of seeds) of Nicotiana alata (grey) and N. longiflora (white). Seeds were obtained from fruits originated of equally pollinated flower sets (5 and 4 respectively) previously subjected to a different number of nectar withdrawals $(\mathrm{X} 2=8.0788, \mathrm{P}=0.005$ and $\mathrm{F}[3,36]=5.84$, $\mathrm{P}<0.0001$, respectively) ordered from the earliest (5) to the last one (1) at the end of the flower lifetime. Boxplot represents: median (medium line), first and third quartile (box), minimum and maximum values (whiskers) and outliers (dots).

\section{Discussion}

The correlation between higher mean nectar amount secreted per flower and the increased number of pollinator visits, with the subsequent higher seed set, was reported for many species (e.g., Zimmerman 1983; Real \& Rathcke 1991; Hodges 1995; Hernandez-Cumplido et al. 2016). This general pattern may suggest that nectar investments within and between species are small or negligible. A recent comparison between nectar-producing and nectarless orchid species showed that pollinators visited more flowers per inflorescence in nectar-producing than in nectarless species (Hobbhahn et al. 2017). Nevertheless, efficiency in pollentransference and seed sets was comparable between these two groups of orchid species (Hobbhahn et al. 2017).

When comparisons on reproductive-resource tradeoffs between nectar secretion and fruit/seed production were focused within the species level, resource investments were experimentally studied only in 10 species (Pleasants \& Chaplin 1983; Zimmerman \& Pyke 1988; Pyke 1991; Ashman \& Schoen 1997; Golubov et al. 2004; Leiss et al. 2004; Ordano \& Ornelas 2005; our data), and the reported responses prevent generalizations. Most of these studies were focused relating nectar production to female fitness, but one study with Asclepias quadrifolia detected trade-off effects when relating resources invested in nectar secretion to male fitness (Pleasants \& Chapin 1983). It is clear that more studies are needed to better understand resource investment trade-offs between nectar production during the pollination process and the consequences for male and female fitness. Consequently, nectar savings during the pollination process through early nectar removal by pollinators can be interpreted as a trade-off between resources secreted by flowers for pollinator attraction and those utilized during fruit and seed maturation.

Results for N. alata and N. longiflora showed that nectar savings during the pollination process through early nectar removal can be related with a higher seed set during fruit development. Nevertheless, when nectar removals were performed later during flower anthesis (i.e., simulated consumption of nectar by pollinators) the consequences on total nectar secretion and female reproductive outputs were lower or negligible. Because these species did not show other alternatives for nectar saving (i.e., nectar resorption at the end of the flower lifetime), effects on female reproduction after the initial secretion period cannot be evidenced (Fig. 5). This general trend for both species can be explained probably because total nectar secretion after repeated removals reached some thresholds (i.e., accumulated values surpass the half of total secretion) during the 2nd or 3rd day of anthesis (according to $N$. alata and N. longiflora, respectively).

The possibility of inhibiting nectar secretion at early stages of flower anthesis after a removal (i.e., after pollinator visits) can occur only during a short period, and it may affect the mating process at the plant population level. These Nicotiana species usually present populations of many flowering individuals (Primo \& Galetto 2001; Roldán et al. 2010). Pollinators face flowers with different amounts of nectar according to the number of previous visits they have received (i.e., the number of nectar removals) and to the variation in nectar production as they age. Low rewarding flowers (because they are recently opened flowers at the initial period of secretion, or because they have received an early visit interrupting nectar secretion, independently of their age) may cause longer-distance flights after a pollinator visit that can promote cross-mating (Hobbhahn et al. 
2017 and references therein). Thus, the effect of resource investment trade-offs between nectar secretion and reproduction will depend on the level at which modularity (flower/fruit, branch or plant) is analyzed (Obeso 2004).

The implications of these resource trade-offs are complex because of the multiple interactions among flowers, pollinators, the physiological events related to the amount of resources involved in pollinator attraction during the anthesis, the pollination process, and the maternal reproductive output (e.g., Obeso 2004; Hobbhahn et al. 2017). Consequently, the gains and costs of resource allocation and nectar investment may vary according to the ecological context. For example, a high nectar investment will be favorable when pollinator availability is low, because it will increase both floral attractiveness and pollination chance. Conversely, when pollinator availability is enough to guarantee pollination and fruit set, those species with flowers that can inhibit nectar secretion (a lower resource investment) early as possible (i.e., nectar saving after a pollinator visit) can be benefited. For example, some species can take advantage when saving part of floral nectar resources and derive them to seed production, compared to those species or populations than cannot modify nectar secretion during the pollination process according to the pollinator environment.

\section{Acknowledgements}

We thank reviewers for useful suggestions and comments that improved this manuscript, Ariel Goldraij and Juan Roldán for share with us their $N$. alata plants for nectar experiments, Lorena Fonseca for her help with $N$. alata data collection, Julia Galetto for English editing services, and the program CAPES (Brazil)-SPU (Argentina) for financial post-graduate student support to LF and FPA. For financial support MS thanks CNPq, FPA CAPES and LG, CT, GG and LDA CONICET, SECyT (UNC), and FONCyT.

\section{References}

Ashman TL, Schoen DJ. 1997. The cost of floral longevity in Clarkia tembloriensis: an experimental investigation. Evolutionary Ecology 11: 289-300.

Bolker BM, Brooks ME, Clark CJ, et al. 2009. Generalized linear mixed models: a practical guide for ecology and evolution. Trends in Ecology \& Evolution 24: 127-135.

Galetto L, Bernardello L. 1993. Nectar secretion pattern and removal effects in three species of Solanaceae. Canadian Journal of Botany 71: 1394-1398.
Galetto L, Bernardello G. 2004. Floral nectaries, nectar production dynamics and chemical composition in six Ipomoea species (Convolvulaceae) in relation to pollinators. Annals of Botany 94: 269-280.

Galetto L, Bernardello G. 2005. Rewards in flowers: Nectar. In: Dafni A, Kevan P, Husband BC. (eds.) Pollination ecology: a practical approach. Cambridge, Enviroquest. p. 156-212.

Golubov J, Mandujano MC, Montaña C, López-Portillo J, Eguiarte LE. 2004. The demographic costs of nectar production in the desert perennial Prosopis glandulosa (Mimosoideae): a modular approach. Plant Ecology 170: 267-275.

Goodspeed TH. 1954. The genus Nicotiana. Chronica Botanica 16: 1-536.

Harder LD, Barrett SCH. 1992. The energy cost of bee pollination for Pontederia cordata (Pontederiaceae). Functional Ecology 6: 226-233.

Hernandez-Cumplido J, Forter B, Moreira X, Heil M, Benrey B. 2016. Induced floral and extrafloral nectar production affect ant-pollinator interactions and plant fitness. Biotropica 48: 342-348.

Hobbhahn N, Johnson SD, Harder LD. 2017. The mating consequences of rewarding vs. deceptive pollination systems: Is there a quantity-quality tradeoff? Ecological Monographs 87: 91-104.

Hodges SA. 1995. The influence of nectar production on hawkmoth behavior, self-pollination, and seed production in Mirabilis multiflora (Nyctaginaceae). American Journal of Botany 82: 197-204.

Ippolito A, Wilson Fernandes G, Holtsford TP. 2004. Pollinator preferences for Nicotiana alata, N. forgetiana, and their F1 hybrids. Evolution 58: 2634-2644.

Leiss KA, Vrieling K, Klinkhamer PGL. 2004. Heritability of nectar production in Echium vulgare. Heredity 92: 446-451.

Obeso JR. 2002. The costs of reproduction in plants. New Phytologist 155: 321-348.

Obeso JR. 2004. A hierarchical perspective in allocation to reproduction from whole plant to fruit and seed level. Perspectives in Plant Ecology, Evolution and Systematics 6: 217-225.

Ordano M, Ornelas JF. 2005. The cost of nectar replenishment in two epiphytic bromeliads. Journal of Tropical Ecology 21: 541-547.

Pleasants JM, Chaplin SJ. 1983. Nectar production rates of Asclepias quadrifolia: causes and consequences of individual variation. Oecologia 59: 232-238.

Primo LM, Galetto L. 2001. Self-and cross-pollen tube growth rates in Nicotiana longiflora (Solanaceae). Boletín de la Sociedad Argentina de Botánica 36: 279-282.

Pyke GH. 1991. What does it cost a plant to produce floral nectar?. Nature 350: 58-59.

R Core Team. 2017. R: A language and environment for statistical computing. Vienna, R Foundation for Statistical Computing. https:// www.R-project.org/

Real LA, Rathcke BJ. 1991. Individual variation in nectar production and its effect on fitness in Kalmia latifolia. Ecology 72: 149-155.

Roldán JA, Quiroga R, Goldraij A. 2010. Molecular and genetic characterization of novel S-RNases from a natural population of Nicotiana alata. Plant Cell Reports 29: 735-746.

Southwick EE. 1984. Photosynthate allocation to floral nectar: a neglected energy investment. Ecology 65: 1775-1779.

Zimmerman M. 1983. Plant reproduction and optimal foraging: experimental nectar manipulations in Delphinium nelsonii. Oikos 41: 57-63.

Zimmerman M, Pyke GH. 1988. Experimental manipulations of Polemonium foliosissimum: effects on subsequent nectar production, seed production and growth. The Journal of Ecology 76: 777-789. 